\title{
The Title Catalog: A Third Dimension
}

The accessibility of the card catalog seems to be inversely proportional to the complexity of its arrangement. A catalog divided into author-title and subject sequences simplifies the filing order of cards and facilitates the use of each catalog. It is argued here that a threeway division into author, title, and subject catalogs will further augment these advantages. In this paper a separation of the title catalog at the University of Wisconsin-Milwaukee library is described and evaluated.

$\mathrm{T}$

HERE IS PRACTICALLY no current literature on dividing the public catalog into separate author, title, and subject alphabets. The pros and cons of separating out the title catalog were discussed prior to World War II, and the topic disappeared from library journals when the controversy over the two-way division of public catalog into author-title and subject catalogs subsided.

The library of the University of Wisconsin-Milwaukee divided its public catalog into author-title and subject catalogs in August 1963; a three-way separation into author, title, and subject catalogs was undertaken in the summer of 1967. The three separate catalogs have been operational since September 1, 1967. The change was accepted overnight by both the patrons and library staff; the benefits of the separation exceeded expectations; and no criticism or complaint has as yet been reported.

Discussion of the merits of the threedimensional public catalog has been reopened for two basic reasons: (a) The-

Mr. Nitecki is Coordinator of Technical Processes in the University of WisconsinMilwaukee. He wishes to acknowledge his appreciation to Mark Gormley, Director of UWM Libraries, for his support of the project and to his colleagues for encouragement and advice. oretically, a combined author-title cata$\log$ is a functional paradox. Author entries, like subject entries, aim at bringing together related works (by author, series, subjects, etc.). The title entry, on the other hand, is a unique feature of each individual work, separating it from any other bibliographical entry. Title arrangement adds a third dimension to the public catalog, which is radically different from the linear, or horizontal, listing by authorship and from the depth, or vertical, grouping by subject. (b) Practically, the verification of library holdings by a known title is a simple activity, easily grasped by patrons unversed in library rules, and a fast and reliable method in a pre-order search by the staff.

\section{Arguments in Favor of Separation}

A consideration to divide the public catalog into separate author, title, and subject catalogs is a logical extension of the arguments once presented in favor of the author-title and subject catalogs. Both the objectives sought at that time and the supporting arguments were already tested in actual use. It is believed that further subdivision will integrate the public catalog even more with the needs of the library. The impact of a fast-growing collection, and the consequent multiplication of catalog cards, will be lessened by a three-part catalog 
since each part will grow proportionally less rapidly. The increasing emphasis on the scholarly collection in an expanding university is reflected in the emerging pattern of searching for a particular book rather than locating books on a particular subject. A separation of author and title catalogs will provide two independent and simultaneous approaches in locating a book, each requiring less search by eliminating irrelevant entries.

Furthermore, the three-part division of the public catalog will break up a complex alphabetical arrangement into three arrangements of decreasing complexity: from the involved subject to the less difficult author, and to the relatively simple title arrangement. This will decrease both filing and retrieval times.

The three-dimensional catalog will also provide for the arrangement of cards by their clearly defined purposes: to provide an index of titles in the title catalog; to list authors in the author catalog; and to group together books on similar subjects. This functional arrangement of catalog cards will allow for an improved economy of use, since it will utilize the distribution of the processes of fast searching for known works and the slower search for unknown titles. It will also reduce the congestion at the card catalogs by providing an easier access to each one of them.

A separate title catalog will be of even greater value to the library staff engaged in checking the library holdings, since the title information on the initial order forms is more often likely to be reliable than the corporate entries in the author catalog. This aspect of the title approach is already successfully utilized in the UWM library by filing LC proof slips by title in the proof slip file.

Instruction in the use of the card catalogs can be reduced to a simple inquiry concerning the traditional "who wrote what on which subject," directing the patron to the appropriate author, title, or subject catalog.
In summary, it seems that little is gained by combining the author and title entries in one catalog. Checking a drawer with a number of similar titles may for some users be more successful than learning to guess at the proper corporate entry for one title. Since the patron is seldom simultaneously interested in a search for the author, title, and subject of the same work, the separate catalogs will contribute to a more direct approach to the information sought. The three-part catalogs provide more spatial flexibility, thus allowing more freedom in the floor arrangement of the catalog room. Finally, a re-filing of the titles back to the author-title catalog is a relatively simple, fast, and inexpensive process. This would allow for easy correction of mistakes not anticipated at the time of separating the catalogs.

\section{The Title Catalog in the UWM LIBRARY}

The development of the separate title catalog in the UWM library was accomplished in three stages: first, the scope of the planned catalog was defined; then the size of the project was estimated by sampling the library collection; and finally the actual project was implemented.

The scope of the title catalog. The policy statement concerning the title catalog was formulated in close cooperation with the coordinator of public services, thus expressing the desire to provide both a simple index for the patrons and an efficient tool for the library staff. The underlying principle in defining the scope of the catalog was to maximize its content first, allowing for the withdrawal of some types of entries later on, if they proved unnecessary in the actual use of the catalog.

Perhaps the single most important decision made was the inclusion of at least one card for each title in the collection. The dilemma of the inclusion of some "common" and insignificant titles is cre- 
Table 1. List of Some Common Titles in the Title Catalog (UWM Library)

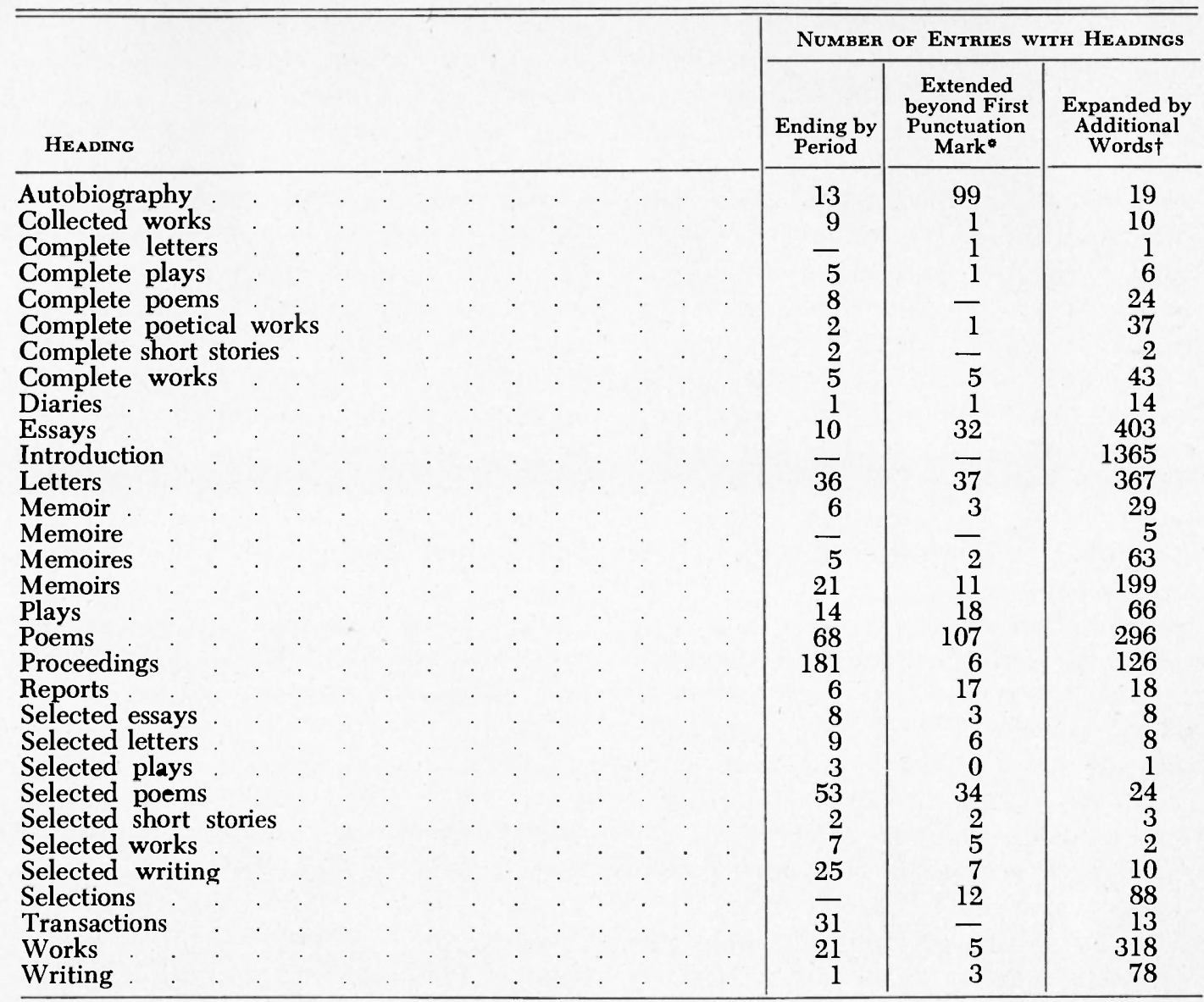

- Includes all punctuation marks except the period (.).

† Many of these entries have titles made by LC.

ated by two convincing but opposing arguments: (a) the more common the title, the easier it is to remember; and (b) the inclusion of all common titles creates a disproportionately large section of identical titles. One solution considered was to exclude the "meaningless" titles. Such a list of exclusions could be compiled gradually, as actual need arises. In each case needed cross references could direct the user from the title to the author catalog.

In the final analysis, the advantages of including all titles outweighed the disadvantages. It was reasoned that the avoidance of exceptions in the coverage of the title catalog would contribute to the simple interpretation of its scope by patrons, while the completeness of the title coverage would significantly increase the reliability of the catalog for searching purposes by the staff. Table 1 lists the number of cards actually filed in each of the more common "meaningless" categories. Each of these larger entries is now separated by guide cards, subdividing each entry by author, and no withdrawal of these cards is at present contemplated.

It is felt that the luxury of making title cards for each entry in the collection can be afforded, since in the bargain the additional luxury is obtained of being able to identify the book in one pass, by 
instructing the student searcher to check the title catalog under the title, exactly as it appears on the title page of the book in hand or as printed in the dealer's catalog. Any discrepancy between the card in the catalog and the title searched is a warning for possible variation in editions. It is known that a significant number of patrons do consult some of these common titles. Once it is determined which of the "meaningless" entries are really meaningless to all the users of the catalog, they will be withdrawn from the title catalog. Now, however, it is easier for a searcher to check one drawer of "poems" under two or three of its sub-arrangements (e.g., under the name of a poet, editor, compiler, or institution) than to walk from one end of the author catalog to the other, if the possible entries happened to be dispersed, between $\mathrm{A}$ and $\mathrm{Z}$. This, together with the simplicity of an unequivocal instruction: "check by title," is worth the extra cards filed under "poems."

The policy concerning the inclusion of other types of entries is less controversial, and some more important decisions are listed here as an illustration of the scope of the title catalog.

As a rule, at least one title card is made for each entry exactly in the form that appears on the title page of the book.

Alternative titles such as binder's titles, caption titles, and catchword titles are not included in the catalog. However, all the titles made by LC, even if they include some of the forms just mentioned, are also added in the catalog. The title catalog is a public record; the inventiveness of the individual cataloger is relative and difficult to anticipate and therefore often "meaningless." The same inventiveness, sanctified by an LC card, renders the title useful and binding.

Additional title cards are also made for transliterated titles, for all forms of changed titles, and for some distinctive titles of the parts of sets.
Title main entries are, of course, made, but they are also duplicated in the author catalog. The concept of the "main entry" is being re-examined, however, and if the concept itself is discarded, the title main entries will also be discarded from the author catalog. In addition, a buff copy of the purchase order is interfiled by title in the title catalog. These slips are replaced by permanent cards after the titles are received and cataloged. A guide card referring the patron to the author catalog is made for each "series made" and filed in the title catalog under the series title. The incomplete open entries and broken sets (i.e., closed entries with gaps in holdings) are placed in a plastic cover with the imprint: "Incomplete-inquire at information desk." The covers are removed after the set is completed. The serials title entries are identified by an additional note directing the user to consult the list of serials holdings which is distributed, in the form of a computerized printout, throughout the library.

Pilot project. In order to estimate the cost and size of the expanded public catalog, a survey of the anticipated changes was conducted at the beginning of summer 1967. Sixteen drawers from selected parts of the shelflist were examined, listing the approximate number of cards in each drawer, the total number of title entries already made, and cards to be added. The survey was based on the assumption that the library would make at least one additional card for each entry.

At the time of the survey, the library's shelflist had a total of 160 drawers, with

TABLE 2. Estimated Expansion of the

Public Catalog in UWM Library by Separating Author and Title Entries

Drawers examined 16

Cards in the drawers

Titles to be added 14,580

Title main entries to be duplicated Total title entries to be added 
Table 3. Reliablility of the Pilot Project

\begin{tabular}{l|c|c|c}
\hline \hline & Estimated Data & Actual Data & Oner-estimation \\
\cline { 5 - 5 } Total number of cards to be added & 36,130 & 31,643 & 4,487 \\
Per cent additions & 20.73 & 18.16 & 2.57 \\
Cost of reproduction & $\$ 957.45$ & $\$ 838.53$ & $\$ 118.92$ \\
\hline
\end{tabular}

approximately the cards distributed about equally among them. In the sample tested, the range varied from 840 to 1,120 cards per drawer, each averaging approximately 6 per cent of the total sample examined.

It was estimated that the public catalog would expand by approximately 16 per cent if all needed titles were made, with an additional increase of 3.8 per cent created by duplication in the author catalog of all title main entries. Since the sixteen drawers examined constituted 10 per cent of the total number of drawers, the projected addition to the title catalog was estimated at 36,130 cards. Assuming the cost of reproducing one card to be $\$ 0.0265$, the total cost of separation (excluding alphabetizing) would amount to $\$ 957.45$. This estimate was, of course, relative to the degree of reliability of the sample tested. To compensate for variable factors (e.g., difference in the thickness of catalog cards) an average of eighty-seven cards per inch was used in all estimates. The sample turned out to be a satisfactory estimate of the percentage expansion of the title catalog, although the sample tested constituted 8.3 per cent of the total shelflist content and not the 10 per cent originally anticipated. As seen in Table 3, the projected expansion of the title catalog by 36,130 additional cards was 2.57 per cent larger than the final number of cards made.

Description of the project. The actual separation of the author-title catalog took place in the two-week recess between the summer and fall semesters of 1967. Sixteen students, supervised by one full-time staff member from the technical processes division, examined, reproduced, and filed cards without interfering with the routine operations of the library, open during that period to the public. The students were assigned to the following stations:

Shelflist: Each drawer was checked for title tracings; titles without title entry were withdrawn.

Retrieval: Main entry cards were pulled from the public catalog, checked against the shelflist cards, and forwarded to the next station. The shelflist cards were stamped with "Title" tracing and refiled in the shelflist catalog. The above two operations were performed in batches of ten cards, thus providing an easy control of cards withdrawn and refiled, keeping the number of cards floating between the catalogs at a minimum and for a very short period of time.

Xeroxing: The main public cards were Xeroxed and immediately refiled in the author catalog. The Xeroxed cards were cut, the holes drilled, and the cards forwarded to the next station.

Preparation: The title on each card was underlined in green ink for filing purposes (UWM library does not raise the title entry), and the cards alphabetized.

Filing: Each card filed was accompanied by a red flag; the flags were removed after the filing was revised.

The average processing times, based on timing three students, each processing 250 cards at one time, were as follows:

Retrieval of cards . . $41.3 \mathrm{sec} . / \mathrm{card}$ Marking . . . . . $12.2 \mathrm{sec}$./card Alphabetizing . . . 14.2 sec./card Filing . . . . . . 56.9 sec./card 
TABLE 4. Estimated Number of Cards

\begin{tabular}{|c|c|c|c|c|c|}
\hline \multirow[b]{2}{*}{ TYPE OF CARD } & \multicolumn{2}{|c|}{$\begin{array}{c}\text { IN AUthor-Title } \\
\text { Catalog } \\
\text { (BEFORE SEPARATion) }\end{array}$} & \multirow{2}{*}{$\begin{array}{c}\begin{array}{c}\text { ADDED } \\
\text { (PLUS } \\
\text { SEPARATED) }\end{array} \\
\text { No. }\end{array}$} & \multicolumn{2}{|c|}{$\begin{array}{c}\text { IN Author \& } \\
\text { TItLE Catalogs } \\
\text { (AFTER SEPARATION) }\end{array}$} \\
\hline & No. & $\begin{array}{l}\text { Per Cent } \\
\text { of Total }\end{array}$ & & No. & $\begin{array}{l}\text { Per Cent } \\
\text { of Total }\end{array}$ \\
\hline $\begin{array}{l}\text { Title entries } \\
\text { Author entries } \\
\text { Total }\end{array}$ & $\begin{array}{l}174,199 \\
283,968 \\
458,167\end{array}$ & $\begin{array}{r}38.1 \\
61.9 \\
100.0\end{array}$ & $\begin{array}{c}31,643 \\
31,643\end{array}$ & $\begin{array}{l}205,842 \\
283,968 \\
489,810\end{array}$ & $\begin{array}{r}42.03 \\
57.97 \\
100.00\end{array}$ \\
\hline
\end{tabular}

The time needed to examine each card for added title entries varied considerably, while the Xeroxing time was determined by the speed of the machine itself. The sets of five cards each could easily be replaced without stopping the Xerox machine.

Over-all estimates. The relative accuracy of estimates is indicated by comparing the total number of titles in the library collection, as reported in the annual report for the year 1966/67 (205,737 titles), and the estimated total number of cards in the title catalog at the time of the completion of the project $(205,842$ cards $)$. These figures exclude United States documents not classified in LC, since no title entries are made for them.

It is estimated that the subject catalog contains approximately 280,000 subject entries plus the average of 170 guide cards per drawer, one guide card for each subject entry used. (UWM library does not raise subject entries.) This figure, however, is approximate, since no attempt was made in this study to determine the ratio of guide cards to subject entries. The estimates of subject cards are not included in any of the tables in this paper.

Estimated cost of transfer in Table 5 is based on a flat wage of $\$ 1.50$ per hour.

TABLE 5. Estimated Cost of Transfer

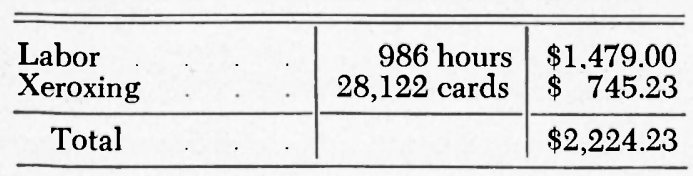

In reality, the cost of labor was substantially reduced by employing a number of students at a lower hourly rate. Furthermore, the separation of the title catalog was performed at the time of transferring the whole public catalog (author-title and subject) to new cabinets. Hence, part of the cost of separating the title catalog, here reported, would be amortized by the over-all cost of transfer.

The cost of Xeroxing, based on a $\$ 0.0265$ unit cost per card, includes material, equipment, and labor at $\$ 1.50$ an hour. The number of cards reproduced $(28,122)$ is 11.1 per cent smaller than the number indicated as added in Table $4(31,643)$ because 3,521 of the "added" cards were from the serial catalog, kept separate till now.

Postscriptum. It is impossible at this time to evaluate accurately the accomplishment achieved at UWM by the separation of the author and title catalogs. Experience with the use of the title catalog since the split indicates that the labor and time involved were a well-invested expenditure. The separate title catalog provides an additional access to the files, by separating different usages; it cuts down the complexities of arrangement, and it speeds routine bibliographic verification of holdings; it makes filing simpler and finding faster. This much is already known. It is also known that the risk involved in attempting to improve library services is an unavoidable price of experimentation which, in turn, is inseparable from progress. 